\title{
Isogeometric Analysis of Euler-Bernoulli Beam Element
}

\author{
Buntara Sthenly Gan ${ }^{1 *}$ \\ ${ }^{I}$ Department of Architecture, College of Engineering, Koriyama, Fukushima 963-8642, Japan \\ * Corresponding author: buntara@arch.ce.nihon-u.ac.jp
}

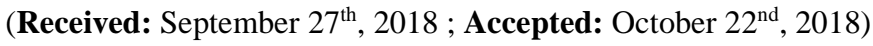

\begin{abstract}
The Isogeometric analysis is a computational geometry based on a series of polynomial functions (Non-Uniform Rational B-Spline, NURBS) which are assembled to represent the exact geometry. In the Isogeometric analysis, the curvature geometry of the beam element can be represented exactly. The conventional beam element can be developed by using the Isogeometric approach which is based on EulerBernoulli principle which is under the assumption that the dimension of the beam cross section is small compared with the length of the beam. The geometric shape of the beam and the shape functions formulation of the element can be formulated by using the Isogeometric approach. This paper highlights the application of the NURBS for the Euler-Bernoulli beam element in the context of finite element analysis. Examples are given to verify the effectiveness of the Isogeometric approach in static and free vibration problems.
\end{abstract}

Keywords: CAD; Euler-Bernoulli beam; Finite element method; Free vibration problem; Isogeometric analysis; NURBS.

\section{Introduction}

Most of the structural components in human-made engineering structures are composed of beams. A beam is a long-block-like structural member whose primary function is to support transverse loading and distribute it to the supports (Fig. 1). The beam resists the transverse loading mainly through bending mechanism. A bending mechanism produces a compressive zone on one side, and the tensile zone on the other side of a beam depends on the loading configurations. In a building structure, a column is also designed based on the classical beam theory with an exception that it can undergo a buckling phenomenon if loaded by an excessive compressive loading. A beam can resist a combination of loading actions. When a beam is supported at two both of its ends where they are permitted to rotate, we call the beam is simply supported. If the beam is fixed at one end and freed at the other end, we call it a cantilever beam.

Rapid advancement in modeling and design is the integration of the CAD-based Isogeometric approach into the finite element method which has been brought up by several authors [1-3]. The integration of the Isogeometric approach with the finite element method in the broad range of modeling contexts has become a popular subject of numerous papers, and many authors have devoted their works to it. Tran et al. [4] formulated the laminated composite plates based on a four-variable refined plate theory. Gan et al. [5-9] analyzed the bending and free vibration of Timoshenko beams using Isogeometric approach. This paper implemented the Isogeometric approach in the finite element for solving the Euler-Bernoulli beam problems. 


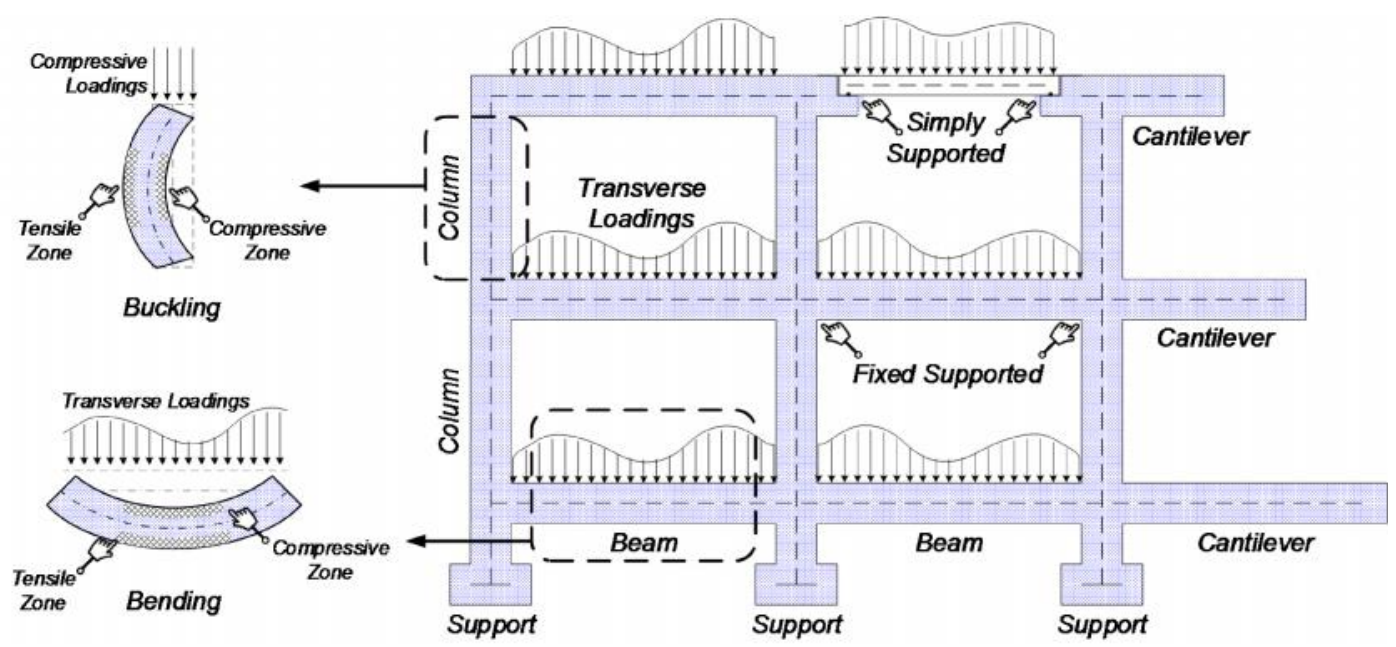

Fig. 1. Beams in a building structure.

\section{Beam element in finite element context}

In the finite element context, a beam is modeled by a line which represents the axis of the beam in the longitudinal direction, or perpendicular to its cross-section (Fig. 2). The line of a beam is called an element. A typical beam element has two nodal points or nodes at both extremities of the line. Each node is given freedom to move or rotate hence we call it a degree-of-freedom (DOF). The term degree is only showing some freedoms to displace or to rotate at the node. The type of freedom at a node can either be a displacement or rotation or a combination between both of them. It is a standard method of the numerical computation; the formulations of beam element in the finite element analysis are presented in the matrices and vectors notations. The simplest and common planar beam element has two nodes at both ends, and each node has a horizontal (tangential) displacement, a vertical (transversal) displacement and a counter-clockwise (CCW) rotation as the DOF. Hence, there are three DOF at each node. Thus six DOF is attributed to a beam element. In vector notation, this six DOF is called as a generalized displacement which is given as follow.

$$
\mathbf{d}=\left[\begin{array}{llllll}
u_{1} & v_{1} & \theta_{1} & u_{2} & v_{2} & \theta_{2}
\end{array}\right]^{T}
$$

The standard beam element formulation is based on the third order polynomial assumption. When the initial shape of a beam is not straight or in the vibration or dynamic analyses problems, higher order polynomial must be assumed. Hence, to increase the accuracy of the calculation, the beam element must be divided into smaller divisions.

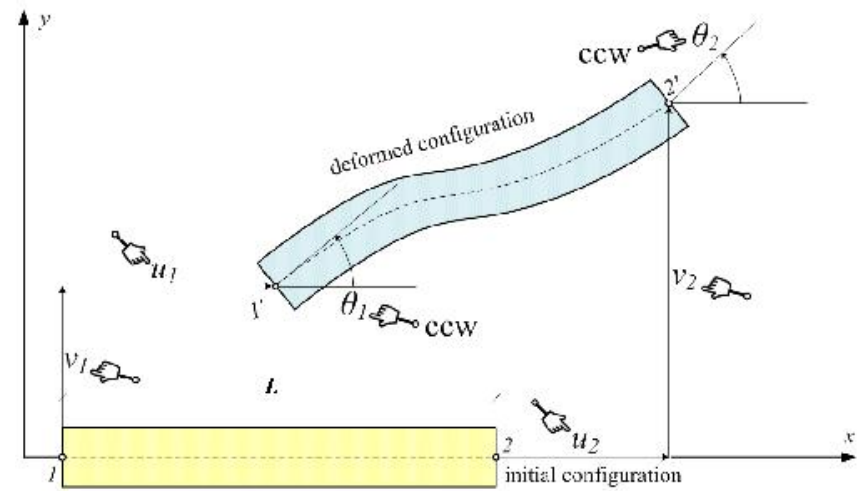

Fig. 2. Beam element in finite element context. 


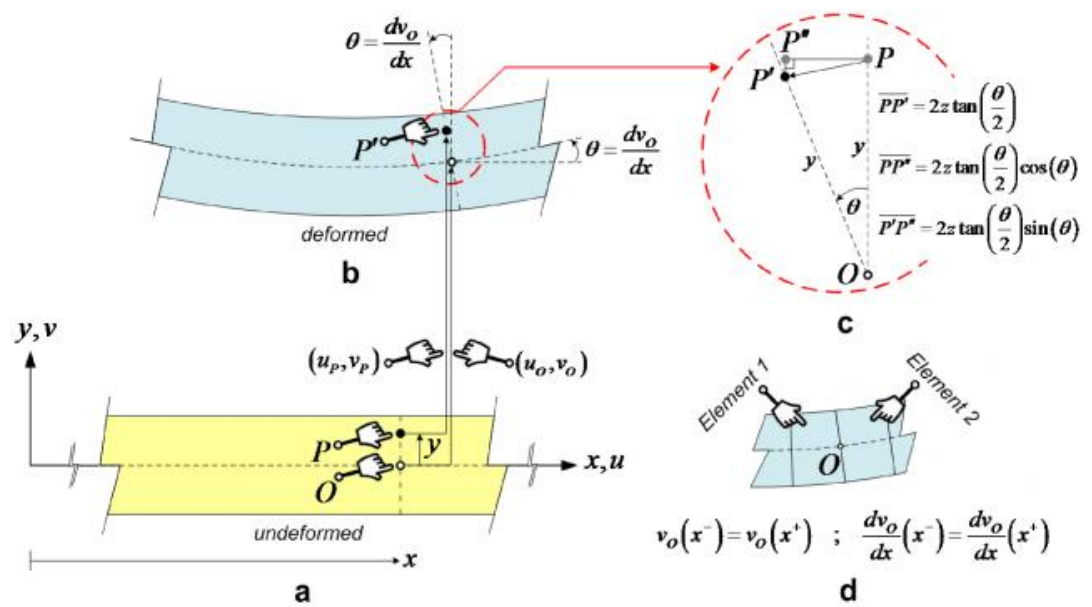

Fig. 3. Conceptual kinematic of the straight Euler-Bernoulli beam element.

\section{Theory of straight Euler-Bernoulli beam element}

The underlying assumption in Euler-Bernoulli beam theory is that the plane of the cross-section remains perpendicular to the beam axis during the deformation. This implies that the rotation of the cross section of the beam is equal to the slope of the beam axis. As illustrated in Fig. 3a-b, this kinematic assumption of a beam leads to the famous beam-bending governing differential equation in which the transverse displacement $v$ is the only variable. Therefore, the continuity conditions between two connected elements at point $O$ require that $v$ and $\frac{d v}{d x}$ are continuous (Fig. 3d). Point $O$ is resting on the axis of the beam.

Under the small displacement assumption, we can apply the following approximations: $\sin (\theta) \approx \tan (\theta) \approx \theta$; and $\cos (\theta) \approx 1 ; \theta^{2} \approx 0$. We can observe from Fig. $3 \mathrm{c}$ that during the deformation of the beam, an arbitrary point $P$ is displaced and rotated by the following assumed displacement fields.

$$
\begin{aligned}
& u_{P}=u_{O}-2 y \tan \left(\frac{\theta}{2}\right) \cos (\theta) \approx u_{O}-2 y \frac{\theta}{2} 1 \approx u_{O}-y \theta \\
& v_{P}=v_{O}+y-y-2 y \tan \left(\frac{\theta}{2}\right) \sin (\theta) \approx v_{O}-2 y \frac{\theta}{2} \theta \approx v_{O} \\
& \theta=\frac{d v_{O}}{d x}
\end{aligned}
$$

where $u_{0}$ and $u_{\mathrm{P}}$ are the horizontal displacements of the point $O$ and $P ; v_{O}$ and $v_{P}$ are the transversal displacements of the point $O$ and $P ; x$ and $y$ are the coordinates of point $P$ before the deformation; $\theta$ is the rotation of the cross section of the beam which is equal to slope the beam at point $O$.

\section{Governing equations of Euler-Bernoulli beam element}

The governing equations of the beam element are derived based on Hamilton's principle. Hamilton's principle is a generalization of the virtual work principle for obtaining the equilibrium equations in the dynamic system. Hence, the governing equations of beam element formulations can be obtained for static and dynamic systems. 
To generalize the displacements and rotation at point $P$, the sub-indexing of the point $O$ in (2) as the reference of deformation will be eliminated. The assumed displacement of the Euler-Bernoulli beam can be rewritten as

$$
\begin{aligned}
& u_{P}=u-y \theta \\
& v_{P}=v
\end{aligned}
$$

from the theory of elasticity, we can obtain the strain component without shear deformation as

$$
\begin{aligned}
& \varepsilon_{x}=\frac{d u_{P}}{d x}=\frac{d u}{d x}-y \frac{d \theta}{d x}=\frac{d u}{d x}-y \frac{d^{2} v}{d x^{2}} \\
& \gamma_{x y}=\frac{d v_{P}}{d x}+\frac{d u_{P}}{d y}=\frac{d v}{d x}-\theta=0 \\
& \therefore \theta=\frac{d v}{d x}
\end{aligned}
$$

The stress component then takes the form

$$
\begin{aligned}
\sigma_{x} & =E \varepsilon_{x}=E\left(\frac{d u}{d x}-y \frac{d^{2} v}{d x^{2}}\right) \\
\tau_{x y} & =0
\end{aligned}
$$

where $E$ is the elastic modulus of the beam.

We can notice that in Eq. (5) only the axial strain component exists in the Euler-Bernoulli beam theory.

The equation of motion is then, can be derived via Hamilton's principle as follow

$$
\delta H=\int_{t_{1}}^{t_{2}}\left(\delta S_{E}-\delta K_{E}-\delta W_{E}\right) d t=0
$$

where,

$$
\begin{aligned}
& \delta H=\text { the variation of total energy } \\
& \delta S_{E}=\text { the variation of beam strain energy } \\
& \delta K_{E}=\text { the variation of beam kinetic energy } \\
& \delta W_{E}=\text { the variation of external work }
\end{aligned}
$$

The strain energy, $S_{E}$, of a beam element with the length $L$ is given as

$$
S_{E}=\frac{1}{2} \int_{0}^{L}\left(\int_{A} \sigma_{x} \varepsilon_{x} d A\right) d x
$$

where $A$ is the cross-section area of the beam.

Substitution of Eq. (4) and Eq. (5) into Eq. (7) gives

$$
S_{E}=\frac{1}{2} \int_{0}^{L}\left(\int_{A}\left(\frac{d u}{d x}-y \frac{d^{2} v}{d x^{2}}\right) E\left(\frac{d u}{d x}-y \frac{d^{2} v}{d x^{2}}\right) d A\right) d x
$$

Applying the variational principle to the above equation, we can have

$$
\delta S_{E}=\int_{0}^{L}\left(\int_{A} E\left(\frac{d u}{d x}-y \frac{d^{2} v}{d x^{2}}\right) \delta\left(\frac{d u}{d x}-y \frac{d^{2} v}{d x^{2}}\right) d A\right) d x
$$


For a symmetric cross-section of the beam with constant $A$ and $E$, we have

$$
\delta S_{E}=\int_{0}^{L}\left\{E A\left(\frac{d u}{d x}\right) \delta\left(\frac{d u}{d x}\right)+E I\left(\frac{d^{2} v}{d x^{2}}\right) \delta\left(\frac{d^{2} v}{d x^{2}}\right)\right\} d x
$$

where,

$$
\begin{aligned}
& A=\int_{A} d A=\iint d y d z \\
& I=\int_{A} y^{2} d A=\iint y^{2} d y d z
\end{aligned}
$$

here, $I$ is the second-order moment of inertia of the beam cross-section.

Applying the integration by part to the first term of Eq. (10),

$$
\int_{0}^{L}\left\{E A\left(\frac{d u}{d x}\right) \delta\left(\frac{d u}{d x}\right)\right\} d x=\left.E A\left(\frac{d u}{d x}\right) \delta u\right|_{B C}-E A \int_{0}^{L}\left(\frac{d^{2} u}{d x^{2}}\right) \delta u d x
$$

where, $B C$ is the boundary conditions of the beam, at $x=0$ and $x=L$, after substitution the above equation becomes

$$
\int_{0}^{L}\left\{E A\left(\frac{d u}{d x}\right) \delta\left(\frac{d u}{d x}\right)\right\} d x=P_{x=L} \delta u_{x=L}-P_{x=0} \delta u_{x=0}-E A \int_{0}^{L}\left(\frac{d^{2} u}{d x^{2}}\right) d x \delta u
$$

where $P_{x=0}, P_{x=L}$ are the axial nodal forces at the beam ends which are given by

$$
\begin{aligned}
& P_{x=0}=\left[E A\left(\frac{d u}{d x}\right)\right]_{x=0} \\
& P_{x=L}=\left[E A\left(\frac{d u}{d x}\right)\right]_{x=L}
\end{aligned}
$$

Applying the integration by part to the second term of Eq. (10),

$$
\int_{0}^{L}\left\{E I\left(\frac{d^{2} v}{d x^{2}}\right) \delta\left(\frac{d^{2} v}{d x^{2}}\right)\right\} d x=\left.E I\left(\frac{d^{2} v}{d x^{2}}\right) \delta v\right|_{B C}-E I \int_{0}^{L}\left(\frac{d^{3} v}{d x^{3}}\right) d x \delta\left(\frac{d v}{d x}\right)
$$

where, $B C$ is the boundary conditions of the beam, at $x=0$ and $x=L$, the above equation becomes

$$
\int_{0}^{L}\left\{E I\left(\frac{d^{2} v}{d x^{2}}\right) \delta\left(\frac{d^{2} v}{d x^{2}}\right)\right\} d x=M_{x=L} \delta\left(\frac{d v}{d x}\right)_{x=L}-M_{x=0} \delta\left(\frac{d v}{d x}\right)_{x=0}-E I \int_{0}^{L}\left(\frac{d^{3} v}{d x^{3}}\right) d x \delta\left(\frac{d v}{d x}\right)
$$

where $M_{x=0}, M_{x=L}$ are the rotational nodal moments at the beam ends which are given by

$$
\begin{aligned}
& M_{x=0}=\left[E I\left(\frac{d^{2} v}{d x^{2}}\right)\right]_{x=0} \\
& M_{x=L}=\left[E I\left(\frac{d^{2} v}{d x^{2}}\right)\right]_{x=L}
\end{aligned}
$$

In the Euler-Bernoulli beam formulation, we notice that there is no shear strain resulted from the formulation because the shear deformation is not considered in the formulation. By further doing the integration by part to the last integral term in Eq. (14), we can have the following 


$$
-\int_{0}^{L}\left\{E I\left(\frac{d^{3} v}{d x^{3}}\right) \delta\left(\frac{d v}{d x}\right)\right\} d x=-Q_{x=L} \delta\left(\frac{d^{3} v}{d x^{3}}\right)_{x=L}+Q_{x=0} \delta\left(\frac{d^{3} v}{d x^{3}}\right)_{x=0}+E I \int_{0}^{L}\left(\frac{d^{4} v}{d x^{4}}\right) d x \delta v
$$

where $Q_{x=0}, Q_{x=L}$ are the transversal nodal forces at the beam ends which are given by

$$
\begin{aligned}
& Q_{x=0}=\left[E I\left(\frac{d^{3} v}{d x^{3}}\right)\right]_{x=0} \\
& Q_{x=L}=\left[E I\left(\frac{d^{3} v}{d x^{3}}\right)\right]_{x=L}
\end{aligned}
$$

The kinetic energy, $K_{E}$, of a beam element with the length $L$ is given as

$$
K_{E}=\frac{1}{2} \int_{0}^{L}\left(\int_{A} \rho\left(\frac{d u}{d t}\right)^{2} d A\right) d x+\frac{1}{2} \int_{0}^{L}\left(\int_{A} \rho\left(\frac{d v}{d t}\right)^{2} d A\right) d x
$$

where $A$ is the cross-section area of the beam. The substitution of Eq. (3) gives,

$$
K_{E}=\frac{1}{2} \int_{0}^{L}\left(\int_{A}\left(\frac{d u}{d t}-y \frac{d \theta}{d t}\right) \rho\left(\frac{d u}{d t}-y \frac{d \theta}{d t}\right) d A\right) d x+\frac{1}{2} \int_{0}^{L}\left(\int_{A}\left(\frac{d v}{d t}\right) \rho\left(\frac{d v}{d t}\right) d A\right) d x
$$

Applying the variational principle to the above equation with constant $A$ and $E$, then the equation become

$$
\delta K_{E}=\rho A \int_{0}^{L}\left(\frac{d u}{d t}\right) \delta\left(\frac{d u}{d t}\right) d x+\rho A \int_{0}^{L}\left(\frac{d v}{d t}\right) \delta\left(\frac{d v}{d t}\right) d x+\rho I \int_{0}^{L}\left(\frac{d \theta}{d t}\right) \delta\left(\frac{d \theta}{d t}\right) d x
$$

Applying the integration by part to the first term of Eq. (18),

$$
\int_{0}^{L}\left\{\rho A\left(\frac{d u}{d t}\right) \delta\left(\frac{d u}{d t}\right)\right\} d x=P_{x=L} \delta u_{x=L}-P_{x=0} \delta u_{x=0}-\rho A \int_{0}^{L}\left(\frac{d^{2} u}{d t^{2}}\right) d x \delta u
$$

where $P_{x=0}, P_{x=L}$ are the axial nodal forces at the beam ends which are given by

$$
\begin{aligned}
& P_{x=0}=\left[\rho A\left(\frac{d u}{d t}\right)\right]_{x=0} \\
& P_{x=L}=\left[\rho A\left(\frac{d u}{d t}\right)\right]_{x=L}
\end{aligned}
$$

Similarly, by applying the integration by part to the second term of Eq. (18),

$$
\int_{0}^{L}\left\{\rho A\left(\frac{d v}{d t}\right) \delta\left(\frac{d v}{d t}\right)\right\} d x=\left.\rho A\left(\frac{d v}{d t}\right) \delta v\right|_{B C}-\rho A \int_{0}^{L}\left(\frac{d^{2} v}{d t^{2}}\right) d x \delta v
$$

where, $B C$ is the boundary conditions of the beam, at $x=0$ and $x=L$, after substitution the above equation becomes

$$
\int_{0}^{L}\left\{\rho A\left(\frac{d v}{d t}\right) \delta\left(\frac{d v}{d t}\right)\right\} d x=P_{x=L} \delta v_{x=L}-P_{x=0} \delta v_{x=0}-\rho A \int_{0}^{L}\left(\frac{d^{2} v}{d t^{2}}\right) d x \delta v
$$

where $Q_{x=0}, Q_{x=L}$ are the transversal nodal forces at the beam ends which are given by 


$$
\begin{aligned}
& Q_{x=0}=\left[\rho A\left(\frac{d v}{d t}\right)\right]_{x=0} \\
& Q_{x=L}=\left[\rho A\left(\frac{d v}{d t}\right)\right]_{x=L}
\end{aligned}
$$

Finally, by applying the integration by part to the third term of Eq. (18),

$$
\int_{0}^{L}\left\{\rho I\left(\frac{d \theta}{d t}\right) \delta\left(\frac{d \theta}{d t}\right)\right\} d x=\left.\rho I\left(\frac{d \theta}{d t}\right) \delta \theta\right|_{B C}-\rho I \int_{0}^{L}\left(\frac{d^{2} \theta}{d t^{2}}\right) d x \delta \theta
$$

where, $B C$ is the boundary conditions of the beam, at $x=0$ and $x=L$, after substitution the above equation becomes

$$
\int_{0}^{L}\left\{\rho I\left(\frac{d \theta}{d t}\right) \delta\left(\frac{d \theta}{d t}\right)\right\} d x=M_{x=L} \delta \theta_{x=L}-M_{x=0} \delta \theta_{x=0}-\rho I \int_{0}^{L}\left(\frac{d^{2} \theta}{d t^{2}}\right) d x \delta \theta
$$

where $M_{x=0}, M_{x=L}$ are the rotational nodal moments at the beam ends which are given by

$$
\begin{aligned}
& M_{x=0}=\left[\rho I\left(\frac{d \theta}{d t}\right)\right]_{x=0} \\
& M_{x=L}=\left[\rho I\left(\frac{d \theta}{d t}\right)\right]_{x=L}
\end{aligned}
$$

The external work, $W_{E}$, of a beam element with the length $L$ is given as

$$
W_{E}=\int_{0}^{L}(p u+q v+m \theta) d x
$$

where, $p, q$, and $m$ are the applied distributed axial force per unit length, transversal force per unit length and moment per unit length, respectively.

Applying the variational principle to the above equation becomes

$$
\delta W_{E}=\int_{0}^{L}(p \delta u+q \delta v+m \delta \theta) d x
$$

In summary, by differentiating the governing equations, the differential equations of motion correspond to generalized displacement can be summarized as

$$
\begin{array}{ll}
\delta u: & -E A \frac{d^{2} u}{d x^{2}}+\rho A \frac{d^{2} u}{d t^{2}}-p=0 \\
\delta v: & +E I \frac{d^{4} v}{d x^{4}}+\rho A \frac{d^{2} v}{d t^{2}}-q=0 \\
\delta \theta: & -E I \frac{d^{3} v}{d x^{3}}+\rho I \frac{d^{2} \theta}{d t^{2}}-m=0
\end{array}
$$

The essential and natural boundary conditions are given as 


$$
\begin{array}{ll}
\delta u: & P=E A \frac{d u}{d x}-\rho A \frac{d u}{d t} \\
\delta v: & Q=-E I \frac{d^{3} v}{d x^{3}}-\rho A \frac{d v}{d t} \\
\delta \theta: & M=E I \frac{d^{2} v}{d x^{2}}-\rho I \frac{d \theta}{d t}
\end{array}
$$

\section{Governing equations in the matrix forms}

The internal strain energy and kinetic energy of the beam at an instant time of $t_{1}=t_{2}$ are given by,

$$
S_{E}=\frac{1}{2} \int_{0}^{L}\left\{E A\left(\frac{d u}{d x}\right)\left(\frac{d u}{d x}\right)+E I\left(\frac{d^{2} v}{d x^{2}}\right)\left(\frac{d^{2} v}{d x^{2}}\right)\right\} d x
$$

and,

$$
K_{E}=\frac{1}{2} \int_{0}^{L}\left\{\rho A\left(\frac{d u}{d t}\right)\left(\frac{d u}{d t}\right)+\rho A\left(\frac{d v}{d t}\right)\left(\frac{d v}{d t}\right)+\rho I\left(\frac{d \theta}{d t}\right)\left(\frac{d \theta}{d t}\right)\right\} d x
$$

with the external work defined by

$$
W_{E}=\int_{0}^{L}\{p u+q v+m \theta\} d x
$$

The shape function is also known as displacement base functions or interpolation functions or generalized displacement. These shape functions have a role in interpolating the displacements or rotation of an arbitrary point along the beam from the nodal displacements and rotations of DOFs at both ends of the beam. The displacements and rotation of any arbitrary point along the beam axis can be interpolated from the nodal general displacement DOF, $\mathbf{d}$, at both nodes of the beam as follow.

$$
\left\{\begin{array}{l}
\mathbf{u} \\
\mathbf{v} \\
\boldsymbol{\theta}
\end{array}\right\}=\left[\begin{array}{cccccc}
N_{u 1} & 0 & 0 & N_{u 2} & 0 & 0 \\
0 & N_{v v 1} & N_{v \theta 1} & 0 & N_{v v 2} & N_{v \theta 2} \\
0 & N_{\theta v 1} & N_{\theta \theta 1} & 0 & N_{\theta v 2} & N_{\theta \theta 2}
\end{array}\right] \cdot\left\{\begin{array}{l}
u_{1} \\
v_{1} \\
\theta_{1} \\
u_{2} \\
v_{2} \\
\theta_{2}
\end{array}\right\}=\mathbf{N} . \mathbf{d}
$$

The derivatives of displacements rotation can be represented by using the nodal general displacement vector $\mathbf{d}$ as

$$
\begin{aligned}
& \frac{d u}{d x}=\left[\begin{array}{ll}
N_{u 1}^{\prime} & N_{u 2}^{\prime}
\end{array}\right]\left\{\begin{array}{l}
u_{1} \\
u_{2}
\end{array}\right\}=\mathbf{N}_{\mathbf{u}}^{\prime}\left\{\begin{array}{l}
u_{1} \\
u_{2}
\end{array}\right\} \\
& \frac{d^{2} v}{d x^{2}}=\left[\begin{array}{llll}
N_{v v 1}^{\prime \prime} & N_{v \theta 1}^{\prime \prime} & N_{v v 2}^{\prime \prime} & N_{v \theta 2}^{\prime \prime}
\end{array}\right]\left\{\begin{array}{l}
v_{1} \\
\theta_{1} \\
v_{2} \\
\theta_{2}
\end{array}\right\}=\mathbf{N}_{\mathbf{v}}^{\prime \prime}\left\{\begin{array}{l}
v_{1} \\
\theta_{1} \\
v_{2} \\
\theta_{2}
\end{array}\right\}
\end{aligned}
$$


By substituting Eq. (32) into Eq. (28), the internal strain energy equation in matrix forms becomes

$$
S_{E}=\frac{1}{2}\left\{\begin{array}{l}
u_{1} \\
v_{1} \\
\theta_{1} \\
u_{2} \\
v_{2} \\
\theta_{2}
\end{array}\right\}^{T} \int_{0}^{L}\left[\begin{array}{l}
\mathbf{N}_{u}^{\prime} \\
\mathbf{N}_{v}^{\prime \prime}
\end{array}\right]^{T}\left[\begin{array}{cc}
E A & 0 \\
0 & E I
\end{array}\right]\left[\begin{array}{l}
\mathbf{N}_{u}^{\prime} \\
\mathbf{N}_{v}^{\prime \prime}
\end{array}\right] d x\left\{\begin{array}{l}
u_{1} \\
v_{1} \\
\theta_{1} \\
u_{2} \\
v_{2} \\
\theta_{2}
\end{array}\right\}
$$

where

$$
\begin{aligned}
\mathbf{N}_{u}^{\prime} & =\left[\begin{array}{llllll}
N_{u 1}^{\prime} & 0 & 0 & N_{u 2}^{\prime} & 0 & 0
\end{array}\right] \\
\mathbf{N}_{v}^{\prime \prime} & =\left[\begin{array}{llllll}
0 & N_{v v 1}^{\prime \prime} & N_{v \theta 1}^{\prime \prime} & 0 & N_{v v 2}^{\prime \prime} & N_{v \theta 2}^{\prime \prime}
\end{array}\right]
\end{aligned}
$$

By substituting Eq. (32) into Eq. (29), the kinetic energy equation in matrix forms becomes

$$
K_{E}=\frac{1}{2}\left\{\begin{array}{c}
\ddot{u}_{1} \\
\ddot{v}_{1} \\
\ddot{\theta_{1}} \\
\ddot{u}_{2} \\
\ddot{v}_{2} \\
\ddot{\theta}_{2}
\end{array}\right\} \int_{0}^{L}\left\{\begin{array}{l}
\mathbf{N}_{u}^{\prime} \\
\mathbf{N}_{v}^{\prime} \\
\mathbf{N}_{\theta}^{\prime}
\end{array}\right\}^{T}\left[\begin{array}{ccc}
\rho A & 0 & 0 \\
0 & \rho A & 0 \\
0 & 0 & \rho I
\end{array}\right]\left\{\begin{array}{l}
\mathbf{N}_{u}^{\prime} \\
\mathbf{N}_{v}^{\prime} \\
\mathbf{N}_{\theta}^{\prime}
\end{array}\right\} d x \cdot\left\{\begin{array}{c}
\ddot{u}_{1} \\
\ddot{v}_{1} \\
\ddot{\theta_{1}} \\
\ddot{u}_{2} \\
\ddot{v}_{2} \\
\ddot{\theta}_{2}
\end{array}\right\}
$$

The substitution of Eq. (31) into Eq. (30), the external works together with the boundary conditions equation in matrix forms become

$$
W_{E}=\left\{\begin{array}{l}
u_{1} \\
v_{1} \\
\theta_{1} \\
u_{2} \\
v_{2} \\
\theta_{2}
\end{array}\right\}^{T}\left\{\begin{array}{c}
N_{u 1} P_{1} \\
N_{v 1} Q_{1} \\
N_{\theta 1} M_{1} \\
N_{u 2} P_{2} \\
N_{v 2} Q_{2} \\
N_{\theta 2} M_{2}
\end{array}\right\}-\left\{\begin{array}{l}
u_{1} \\
v_{1} \\
\theta_{1} \\
u_{2} \\
v_{2} \\
\theta_{2}
\end{array}\right\}^{T} \int_{0}^{L}\left\{\begin{array}{c}
N_{u 1} p \\
N_{v 1} q \\
N_{\theta 1} m \\
N_{u 2} p \\
N_{v 2} q \\
N_{\theta 2} m
\end{array}\right\} d x
$$

The theory of minimum potential energy can be used conveniently to develop the finite element formulation for a straight Euler-Bernoulli beam to obtain the equilibrium equation in matrix form.

$$
\begin{aligned}
& \Pi=\frac{1}{2} \mathbf{d}^{T} \mathbf{K d}-\frac{1}{2} \ddot{\mathbf{d}}^{T} \mathbf{M} \ddot{\mathbf{d}}-\mathbf{d}^{T} \mathbf{f} \\
& \frac{\partial \Pi}{\partial \mathbf{d}^{T}}=\mathbf{K} \mathbf{d}-\mathbf{M} \ddot{\mathbf{d}}-\mathbf{f}=\mathbf{0}
\end{aligned}
$$

The stiffness, mass matrices, and loading vector are given by

$$
\mathbf{K}=\int_{0}^{L}\left[\begin{array}{l}
\mathbf{N}_{u}^{\prime} \\
\mathbf{N}_{v}^{\prime \prime}
\end{array}\right]^{T}\left[\begin{array}{cc}
E A & 0 \\
0 & E I
\end{array}\right]\left[\begin{array}{l}
\mathbf{N}_{u}^{\prime} \\
\mathbf{N}_{v}^{\prime \prime}
\end{array}\right] d x
$$




$$
\begin{aligned}
\mathbf{M} & =\int_{0}^{L}\left\{\begin{array}{l}
\mathbf{N}_{u}^{\prime} \\
\mathbf{N}_{v}^{\prime} \\
\mathbf{N}_{\theta}^{\prime}
\end{array}\right\}^{T}\left[\begin{array}{ccc}
\rho A & 0 & 0 \\
0 & \rho A & 0 \\
0 & 0 & \rho I
\end{array}\right]\left\{\begin{array}{l}
\mathbf{N}_{u}^{\prime} \\
\mathbf{N}_{v}^{\prime} \\
\mathbf{N}_{\theta}^{\prime}
\end{array}\right\} d x \\
\mathbf{f} & =\left\{\begin{array}{c}
N_{u u 1} P_{1} \\
N_{v v 1} Q_{1} \\
N_{\theta \theta 1} M_{1} \\
N_{u u 2} P_{2} \\
N_{v v 2} Q_{2} \\
N_{\theta \theta 2} M_{2}
\end{array}\right\}+\int_{0}^{L}\left\{\begin{array}{c}
N_{u u 1} p \\
N_{v v 1} q \\
N_{\theta \theta 1} m \\
N_{u u 2} p \\
N_{v v 2} q \\
N_{\theta \theta 2} m
\end{array}\right\} d x
\end{aligned}
$$

\section{Isogeometric approach to beam element}

The isogeometric approach is pioneered by several researchers [1-3] where the NURBS curves are used to represent the real geometry and direct mesh modeling in the finite element context. The NURBS with its informative geometrical information which is usually created in the CAD environment can be transported seamlessly into the finite element formulation. In the formulation of the NURBS-based element, the shape function which is a fundamental component in element formulation can also be constructed straightforwardly

A straight beam element is considered as a one-dimensional problem. In the isogeometric approach, the coordinates of the geometry of the beam can be represented by using the NURBS curve as follow,

$$
x=\sum_{i=0}^{n} S_{i, p}(\xi) P_{x i} \quad-1 \leq \xi \leq 1
$$

where

$$
S_{i, n}(\xi)=\frac{N_{i, p}(\xi) w_{i}}{\sum_{j=0}^{n} N_{j, p}(\xi) w_{j}} \quad w_{i}>0
$$

In isogeometric approach, the classical shape functions, $\mathbf{N}_{u / v}(\xi)$, and their derivatives of the Euler-Bernoulli straight beam element in Eq. (31) are substituted by the rational basis functions for the B-spline curve, $S_{i, p}(\xi)$ from Eq. (40) and the derivative of the rational basis function $S_{i, n}^{k}(\xi)$ can be computed.

The displacements and rotation of any arbitrary point along the beam can be calculated from the general nodal displacement of the beam element by using the shape functions from B-spline curve which are given as

$$
u=\sum_{i=0}^{n u} S_{i, p u}(\xi) u_{i} \quad ; \quad v=\sum_{i=0}^{n v} S_{i, p v}(\xi) v_{i} \quad ; \quad \theta=\frac{d v}{d \xi}=\sum_{i=0}^{n v} S_{i, p v}^{1}(\xi) \theta_{i}
$$

Depends on the order of the $p$-th degree polynomial and knot vector $(\Xi)$ being selected, we need to prepare the corresponding number of control points $(C P)$ required. The increasing of control points being used to construct the NURBS curve will increase the degree-of-freedom of the beam as illustrated in Fig. 4.

The classical Euler-Bernoulli beam shape functions were incomplete polynomials due to the assumption of a cross-section of the beam remain perpendicular to the beam axis, where the 
derivative of vertical displacement assumption was used for the slope of the beam. However, in the isogeometric approach using the NURBS, a complete series of rational basis B-spline functions are necessary to compose a function of the order $p$-th degree curve.

Therefore, the series of rational basis B-spline functions of the NURBS curve cannot be used directly to compose the shape functions of the Euler-Bernoulli beam. To apply the rational basis B-spline functions for the vertical displacement and rotational for the Euler-Bernoulli beam shape functions, selective basis functions $B_{i, p v}\left(\xi_{j}\right)$ which are taken from the series of rational basis Bspline functions of the NURBS that will be customized and used to fit the classical shape functions.

The selective basis functions are given as

$$
\begin{aligned}
& B_{0, p v}\left(\xi_{j}\right)=S_{0, p v}\left(\xi_{j}\right)+S_{1, p v}\left(\xi_{j}\right) \\
& B_{1, p v}\left(\xi_{j}\right)=S_{2, p v}\left(\xi_{j}\right)+S_{3, p v}\left(\xi_{j}\right) \\
& B_{2, p v}\left(\xi_{j}\right)=\frac{L}{3} S_{1, p v}\left(\xi_{j}\right) \\
& B_{3, p v}\left(\xi_{j}\right)=-\frac{L}{3} S_{2, p v}\left(\xi_{j}\right)
\end{aligned}
$$

By replacing the with shape function $\mathbf{N}_{u / v}(\xi)$ by $\mathbf{B}_{p v}(\xi)$, the stiffness; mass; and loading vector of the beam element can be constructed by using Eq. (37-39).

\section{Numerical Examples}

The verification of the Isogeometric analysis for the beam element is conducted for static and free vibration dynamic problems.

\subsection{Static problems of Euler-Bernoulli beams}

Consider a cantilever beam as shown in Fig. 5 . The beam has geometry and property depicted in the figure. The degree of polynomial used in the NURBS functions are $p_{u}=1$ and $p_{v}=3$.

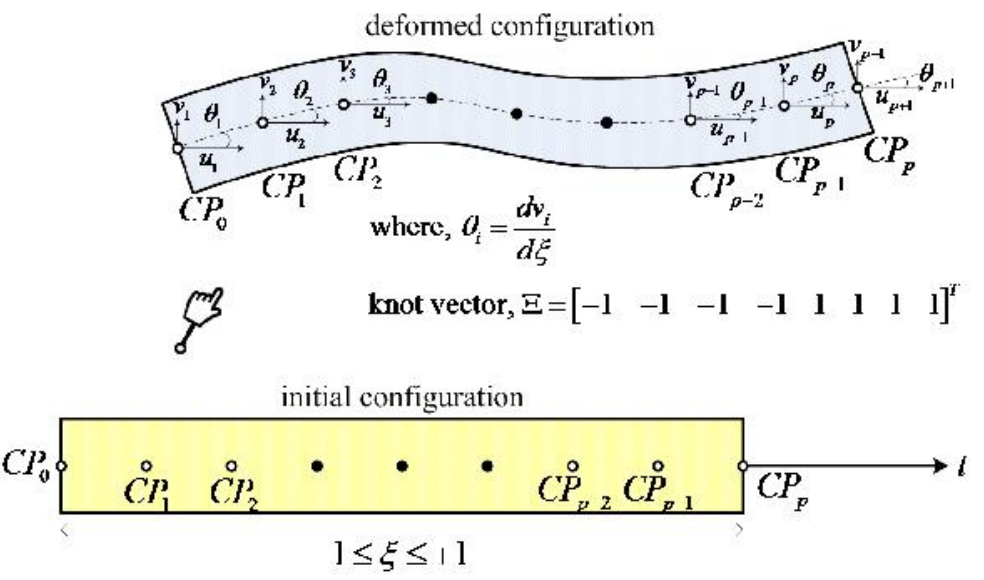

Fig. 4. Conceptual Euler-Bernoulli beam element in isogeometric approach. 
The beam is subjected to various loadings conditions. One element with four Gauss integration points is used in the analysis. The results of static analyses are tabulated in Table 1.

Table 1. Results of static analyses of a cantilever beam subjected to various loadings

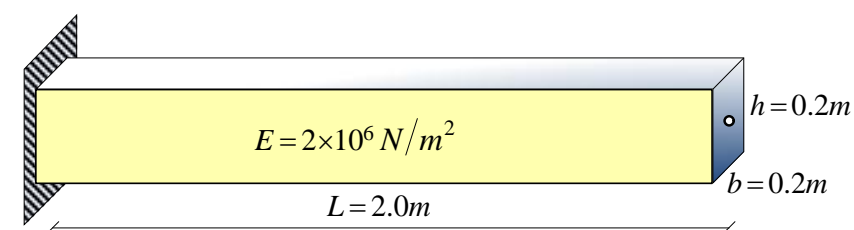

Fig. 5. Cantilever Euler-Bernoulli straight beam under various loadings.

\begin{tabular}{|c|c|c|c|c|}
\hline \multirow[t]{2}{*}{ Type of loading } & \multicolumn{2}{|c|}{$\begin{array}{l}\text { Deflection at the free end } \\
\qquad\left(10^{-3} \mathrm{~m}\right)\end{array}$} & \multicolumn{2}{|c|}{$\begin{array}{l}\text { Rotation at the free end } \\
\qquad\left(10^{-3} \mathrm{rad}\right)\end{array}$} \\
\hline & Analysis & Theory & Analysis & Theory \\
\hline $\begin{array}{l}\text { Concentrated Load } \\
P_{\mathrm{y}} \text { at free end }(1 \mathrm{~N})\end{array}$ & -10.00 & $v_{\max }=-\frac{P L^{3}}{3 E I}=-10.00$ & -7.500 & $\theta_{\max }=-\frac{P L^{2}}{2 E I}=-7.500$ \\
\hline $\begin{array}{l}\text { Bending Moment } \\
M_{\mathrm{z}} \text { at the free end }(1 \mathrm{Nm})\end{array}$ & 7.500 & $v_{\max }=\frac{M L^{4}}{2 E I}=7.500$ & 7.500 & $\theta_{\max }=\frac{M L}{E I}=7.500$ \\
\hline $\begin{array}{l}\text { Distributed Load } \\
q_{\mathrm{y}} \text { along the } \operatorname{span}(1 \mathrm{~N} / \mathrm{m})\end{array}$ & 7.500 & $v_{\max }=\frac{q L^{4}}{8 E I}=7.500$ & 5.000 & $\theta_{\max }=\frac{q L^{3}}{6 E I}=5.000$ \\
\hline
\end{tabular}

The beam is subjected to several loadings conditions. One element with four Gauss integration points is used in the analysis. The results of static analyses are given in Table 2. Next, consider a simply supported beam as shown in Fig. 6 . The beam has geometry and property depicted in the figure.

Table 2. Results of static analyses of a simply supported beam subjected to various loadings

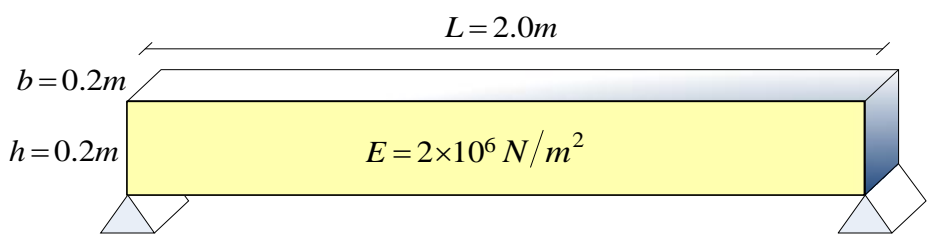

Fig. 6. Simply supported Euler-Bernoulli straight beam under various loadings.

\begin{tabular}{|c|c|c|c|c|}
\hline \multirow[t]{2}{*}{ Type of loading } & \multicolumn{2}{|c|}{$\begin{array}{l}\text { Deflection at the free end } \\
\qquad\left(10^{-3} \mathrm{~m}\right)\end{array}$} & \multicolumn{2}{|c|}{$\begin{array}{l}\text { Rotation at the free end } \\
\left(10^{-3} \mathrm{rad}\right)\end{array}$} \\
\hline & Analysis & Theory & Analysis & Theory \\
\hline $\begin{array}{l}\text { Concentrated Load } \\
P_{\mathrm{y}} \text { at mid-span }(1 \mathrm{~N})\end{array}$ & -0.6250 & $v_{\max }=-\frac{P L^{3}}{48 E I}=$ & \pm 0.9375 & $\theta_{\max }= \pm \frac{P L^{2}}{16 E I}= \pm 0.9375$ \\
\hline $\begin{array}{l}\text { Bending Moment } \\
M_{\mathrm{z}} \text { at mid-span }(1 \mathrm{Nm})\end{array}$ & 0.0 & 0.0 & -0.3125 & $\theta_{\max }=-\frac{M L}{24 E I}=-0.3125$ \\
\hline
\end{tabular}


Distributed Load

$q_{\mathrm{y}}$ along the span $(1 \mathrm{~N} / \mathrm{m})$

$$
-0.78125 v_{\max }=-\frac{5 q L^{4}}{384 E I}= \pm 1.250 \quad \theta_{\max }= \pm \frac{q L^{3}}{24 E I}= \pm 1.250
$$

Results which are listed in Table 1. and Table 2. show excellent agreement with those the classical beam theory equations.

\subsection{Free vibration problems of Euler-Bernoulli beams}

Consider the simply supported beam shown in Fig 6. as a free vibration problem.

The beam with the length $L=10 \mathrm{~m}$, the rectangular cross-section with width $b=1.5 \mathrm{~m}$ and depth $h=2 \mathrm{~m}$. The elastic modulus $E=1 \mathrm{~N} / \mathrm{m}^{2}$, the density $\rho=1 \mathrm{~kg} / \mathrm{m}^{3}$. Four Gauss integration points are necessary to integrate the mass matrix. The degree of polynomial used in the NURBS functions are $p_{u}=1$ and $p_{v}=3$.

The free vibration problems were analyzed by using two, three and four elements sub-division are given in Table 3 . The results are compared with the exact values $\left(\omega_{E B-n}\right)$ from the $n^{\text {th }}$ mode of free vibration of Euler-Bernoulli beam theory which is defined by,

Table 3. Free vibration results of the first six normalized natural frequencies of simply supported Euler-Bernoulli beam

\begin{tabular}{|c|c|c|c|c|}
\hline \multirow{2}{*}{$\begin{array}{c}\text { Mode } \\
\text { Number } \\
n\end{array}$} & & \multicolumn{3}{|c|}{$\frac{\omega_{n}}{\omega_{E B-n}}$} \\
\hline & $\omega_{E B-n}$ & 2 elements & 3 elements & 4 elements \\
\hline 1 & 20.1462 & 1.0039 & 1.0008 & 1.0003 \\
\hline 2 & 80.5850 & 1.1099 & 1.0118 & 1.0039 \\
\hline 3 & 181.3162 & 1.2399 & 1.1099 & 1.0183 \\
\hline 4 & 322.3399 & 1.2716 & 1.1609 & 1.1099 \\
\hline 5 & 503.6561 & --- & 1.3294 & 1.1291 \\
\hline 6 & 725.2648 & --- & 1.2716 & 1.2399 \\
\hline
\end{tabular}

From Table 3., we can observe that by increasing the number of the element up to only four subdivisions, we can get better results with the first six frequency modes of the Euler-Bernoulli straight beam free vibration problems.

\section{Conclusions}

An Euler-Bernoulli beam element formulation is developed by using the Isogeometric approach and used to evaluate the vibration behaviors of various straight beam structures. The efficiency and accuracy of the isogeometric approach are verified by exact solutions through numerical examples for static and dynamic problems. From the numerical results, the following concluding remarks can be drawn, 
The isogeometric approach is bridging the NURBS line created by CAD, then solved by the finite element analysis directly.

\section{References}

[1] Hughes, T.J.R., Cottrell, J.A., Bazilevs, Y. (2005). Isogeometric analysis: CAD, finite elements, NURBS, exact geometry and mesh refinement. Computer Methods in Applied Mechanics and Engineering, 194(39-41), 4135-4195.

[2] Cottrell, J.A., Bazilevs, Y., Hughes, T.J.R., (2009), Isogeometric Analysis: Towards Integration of CAD and FEA, New York: Wiley.

[3] Gan, B.S. (2018), An Isogeometric Approach to Beam Structure, Switzerland: Springer International Publishing.

[4] Tran, L.V., Thai, C.H., Le, H.T., Gan, B.S., Lee, J., Nguyen, X.H. (2014). Isogeometric analysis of laminated composite plates based on a four-variable refined plate theory. Engineering Analysis with Boundary Elements, Elsevier, 47, 68-81.

[5] Gan, B.S., Nguyen, D.K., Han, A., Alisjahbana, S.W. (2018). DOF Condensation of Thick Curved Beam Element Formulated by Isogeometric Approach. International Conference on Advances in Computational Mechanics, Springer, 545-559.

[6] Gan, B.S., Han, A. (2018). Two Nodes Cusp Geometry Beam Element by using Condensed IGA. The 4th International Conference on Sustainable Civil Engineering Structures and Construction Materials (SCESCM 2018), Conference Proceeding.

[7] Gan, B.S. (2017), Isogeometric Analysis for Beam Element. The 2nd International Joint Conference on Advanced Engineering and Technology (IJCAET 2017), Conference Proceeding.

[8] Gan, B.S., Nguyen, D.K., Han, A., Alisjahbana, S.W. (2017). DOF Condensation of thick curved beam element formulated by Isogeometric Approach. The International Conference on Advances in Computational Mechanics, ACOME 2017, Conference Proceeding.

[9] Gan, B.S. (2017), A Two-node Beam Element by using Isogeometric Approach, The 14th U.S. National Congress on Computational Mechanics, Conference Proceeding. 